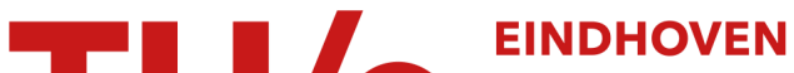 UNIVERSITY OF TECHNOLOGY
}

\section{The dual risk model with dividends taken at arrival}

Citation for published version (APA):

Boxma, O., \& Frostig, E. (2018). The dual risk model with dividends taken at arrival. Insurance: Mathematics and Economics, 83, 83-92. https://doi.org/10.1016/j.insmatheco.2018.09.005

\section{Document license:}

TAVERNE

DOI:

10.1016/j.insmatheco.2018.09.005

Document status and date:

Published: 01/11/2018

\section{Document Version:}

Publisher's PDF, also known as Version of Record (includes final page, issue and volume numbers)

\section{Please check the document version of this publication:}

-A submitted manuscript is the version of the article upon submission and before peer-review. There can be important differences between the submitted version and the official published version of record. People interested in the research are advised to contact the author for the final version of the publication, or visit the $\mathrm{DOI}$ to the publisher's website.

- The final author version and the galley proof are versions of the publication after peer review.

- The final published version features the final layout of the paper including the volume, issue and page numbers.

Link to publication

\section{General rights}

Copyright and moral rights for the publications made accessible in the public portal are retained by the authors and/or other copyright owners and it is a condition of accessing publications that users recognise and abide by the legal requirements associated with these rights.

- Users may download and print one copy of any publication from the public portal for the purpose of private study or research.

- You may not further distribute the material or use it for any profit-making activity or commercial gain

- You may freely distribute the URL identifying the publication in the public portal.

If the publication is distributed under the terms of Article 25fa of the Dutch Copyright Act, indicated by the "Taverne" license above, please follow below link for the End User Agreement:

www.tue.nl/taverne

Take down policy

If you believe that this document breaches copyright please contact us at:

openaccess@tue.nl

providing details and we will investigate your claim. 


\title{
The dual risk model with dividends taken at arrival
}

\author{
Onno Boxma ${ }^{a, *}$, Esther Frostig ${ }^{b}$ \\ ${ }^{a}$ Department of Mathematics and Computer Science, Eindhoven University of Technology, P.O. Box 513, 5600 MB Eindhoven, The Netherlands \\ b Department of Statistics, Haifa University, Haifa, Israel
}

\section{A R T I C L E I N F O}

\section{Article history:}

Received December 2017

Received in revised form September 2018

Accepted 17 September 2018

Available online 22 September 2018

\section{Keywords:}

Dual risk model

Time to ruin

Dividend

$M / G / 1$ queue

Busy period

\begin{abstract}
A B S T R A C T
We consider the dual risk model with special dividend or tax payments: If an arriving gain finds the surplus above a barrier $b$ or if it would bring the surplus above that level, a certain part of the gain is paid as dividends or taxes. We obtain expressions for the joint Laplace-Stieltjes transform of the time to ruin and the amount of dividends paid until ruin, and for the expected discounted dividend paid until ruin. We consider the case where the dividend paid from each gain is a general function of the gain. More explicit results are obtained when the dividend is a given percentage of the gain amount.
\end{abstract}

(c) 2018 Elsevier B.V. All rights reserved.

\section{Introduction}

This paper is devoted to the analysis of dividend policies in the compound Poisson dual risk model. Since the pioneering work of De Finetti, many types of dividend policies have been studied for the classical Cramér-Lundberg model, i.e., for risk processes modeled as a Lévy process without positive jumps. In the barrier dividend strategy all the premium inflow received while the process is above the barrier is paid as dividends. Under some conditions, this policy was proven to be optimal for the classical risk model. Another dividend policy is the threshold strategy, where dividends are paid at a fixed rate smaller than the premium rate, when the surplus is above a given horizontal threshold. This policy was proven to be optimal under some conditions when the dividend rate is bounded from above. For a comprehensive review on the dividends strategies, see Avanzi (2009) and Albrecher and Thonhauser (2009).

Another process that has been studied in the actuarial literature is the dual risk model. This is a Lévy process without negative jumps. It describes the surplus of a company with fixed expense rate and occasional gains. Examples are pharmaceutical, petroleum, or R\& D companies. Since Avanzi et al. (2007) various performance measures of the dual risk model have been studied. Avanzi et al. (2007) and Avanzi (2009) considered the optimal dividend policy for the compound Poisson dual risk model with and without perturbation of Brownian motion and proved that the policy that maximizes the expected present value of the dividends is the barrier strategy, i.e., all the overflow above a barrier is paid as

\footnotetext{
* Corresponding author.

E-mail addresses: o.j.boxma@tue.nl (0. Boxma), frostig@stat.haifa.ac.il (E. Frostig).
}

dividends. The barrier strategy is proven to be optimal for the more general spectrally positive Lévy process in Bayraktar et al. (2013) as well as for the dual risk process with terminal cost (Yin and Wen, 2013). Under the threshold policy no dividends are paid when the surplus is below a given horizontal threshold and dividends are paid continuously at a constant rate when the surplus is above that barrier. $\mathrm{Ng}$ (2009) studied the threshold dividend strategy for the dual risk model. Yin et al. (2014) proved that the threshold policy is optimal under the restriction that the dividends are absolutely continuous and the dividend rate is bounded from above. When dividends can be either absolutely continuous with bounded rate or given as a lump amount, the optimal policy is a combination of the threshold policy and the barrier strategy (Avanzi et al., 2017).

In this paper we consider the compound Poisson dual risk model with the following dividend policy: Above a given horizontal barrier a certain part of each gain (or from the part of a gain which brings the surplus above the barrier) is paid out as dividends or as taxes. Thus it differs from the barrier strategy since not all the overflow above the barrier is paid as dividends and it differs from the threshold policy since dividends are not paid continuously. As an example consider R\&D, pharmaceutical or petroleum companies, where whenever there is a gain and the surplus of the company is high it gives some bonus or dividends to its employees or shareholders from each gain. Alternatively, especially in the petroleum industry, a company might pay tax from each gain to the regulator. Our main results are expressions for the joint LaplaceStieltjes transform of the time to ruin and the amount of dividends, and for the expected discounted dividends until ruin.

The paper is organized as follows. Section 2 contains a model description and some results about scale functions that will be used later on. Section 3 is devoted to the case that a fixed proportion of the gain above a certain threshold is paid out as dividend, while 
Section 4 considers a general function of the gain. In both cases, we derive expressions for the joint Laplace-Stieltjes transform of the time to ruin and the total dividend until ruin; we also discuss the expected discounted dividends.

\section{Preliminaries}

\subsection{The model}

Let $U(t)$ be the surplus process of the dual risk model without dividend payments:

$U(t)=U(0)-c t+\sum_{i=1}^{N(t)} Y_{i}$

where $c>0, N(t)$ is a Poisson process with rate $\lambda$ and $Y_{i}, i=$ $1,2, \ldots$, are i.i.d. positive random variables distributed as $Y$ with distribution $G$ and Laplace-Stieltjes transform $\mathcal{L}_{Y}(\cdot)$. $U(t)$ can also represent the work in process in an $\mathrm{M} / \mathrm{G} / 1$ queue or the inventory level in a storage model or dam model with occasional inflow and a constant demand rate. It is well-known that

$\mathbb{E}\left[e^{-\theta(U(t)-U(0))}\right]=e^{t \psi(\theta)}$,

where

$\psi(\theta)=c \theta-\lambda+\lambda \mathcal{L}_{Y}(\theta)$

$\psi(\theta)$ is the Laplace exponent of the process. Let $\Phi(\theta)$ be the biggest solution to

$\psi(\theta)=0$.

It is well-known that when $\psi^{\prime}(0) \geq 0$ then $\Phi(0)=0$, otherwise $\Phi(0)>0$. When $\psi^{\prime}(0) \geq 0$ the busy period in the $\mathrm{M} / \mathrm{G} / 1$ queue is finite with probability 1 , or in the dual risk model ruin occurs with probability 1 . Otherwise the busy period can be infinite with positive probability or equivalently, the ruin probability is less than 1.

We consider a horizontal barrier $b>0$. From each gain of size $x$ that occurs while the process is above $b$ or from each overshoot of size $x$ of a gain that brings the surplus above $b$, a dividend of size $I(x)$ is paid. We assume that $0<I(x)<x$.

Thus above $b$ the process behaves as $\mathbb{U}$, where

$d \mathbb{U}(t)=-c d t+d S(t)$,

where $S(t)=\sum_{i=1}^{N(t)}\left(Y_{i}-I\left(Y_{i}\right)\right)$. Throughout the paper we assume that $c=1$, and we denote by $\mathcal{L}_{A}$ the Laplace-Stieltjes transform of a random variable $A$.

The Laplace exponent of $\mathbb{U}(t)$ is $\psi_{1}(\theta)$, where

$\psi_{1}(\theta)=\theta-\lambda+\lambda \mathcal{L}_{Y-I(Y)}(\theta)$.

Let $\Phi_{1}$ be the inverse of $\psi_{1}$. We denote by $X_{1}$ the spectrally negative risk process with $d X_{1}(t)=-d \mathbb{U}(t)$. Let $\mathcal{U}$ be the net surplus process, i.e., after dividends payout. Our aim is to obtain the Laplace-Stieltjes transform of the total dividends and the expected discounted dividend paid until ruin. We consider three versions of the above policy:

v1. When the initial surplus $u$ is bigger than $b$ then $(1-\alpha)(u-b)$ is paid immediately as dividends at time 0 . Otherwise dividends are paid as described above.

v2. When the initial surplus $u$ is bigger than $b$ then no dividends are paid at time zero. Otherwise dividends are paid as described above.

v3. No dividends are paid at time 0 . After time 0 no dividends are paid from the overshoot above $b .(1-\alpha)$ of each gain that arrives when the surplus is above $b$, is paid as dividends.

We shall, in short, refer to models v1, v2 and v3 when considering our model with these three policy versions.

\subsection{Scale function}

In this subsection we review some fundamental results for the exit times for spectrally negative Lévy processes in terms of scale functions; these results are used in the rest of the paper.

Let $X(t)$ be a spectrally negative Lévy process, and more specifically,

$X(t)=X(0)+t-\sum_{i=1}^{N(t)} Y_{i}$.

In our context $X(t)=b-U(t)$, thus $X(0)=b-U(0)$. Hence, cf. (2.2),

$\mathbb{E}\left[e^{\theta(X(t)-X(0))}\right]=e^{\psi(\theta) t}$,

where $\psi$ is as in Eq. (2.3).

For the following notations and definitions we refer the reader to Chapter 8 in Kyprianou (2006). The $q$-scale function, $W^{(q)}(x)$, is the unique right-continuous function whose Laplace transform is given by

$\int_{0}^{\infty} e^{-\beta x} W^{(q)}(x) d x=\frac{1}{\psi(\beta)-q}, \quad$ for $\beta>\Phi(q)$.

We also define the function $Z^{(q)}(x, \theta)$ by

$Z^{(q)}(x, \theta)=e^{\theta x}\left(1-(\psi(\theta)-q) \int_{0}^{x} e^{-\theta y} W^{(q)}(y) d y\right)$,

(see also Eqs. (4)-(5) in Albrecher et al., 2016). For $\theta=0$ we get the function $Z^{(q)}(x)$,

$Z^{(q)}(x)=1+q \int_{0}^{x} W^{(q)}(y) d y$.

Let $\tau_{b}^{+}=\inf \{t: X(t)=b\}$ and let $\tau_{a}^{-}=\inf \{t: X(t) \leq a\}$. Then for $0 \leq x<b$ the following holds (by $\mathbb{E}_{x}$ we denote the conditional expectation given that $X(0)=x)$ :

$\mathbb{E}_{0}\left[e^{-q \tau_{b}^{+}} 1_{\left\{\tau_{b}^{+}<\infty\right\}}\right]=e^{-\Phi(q) b}$,

$\mathbb{E}_{x}\left[e^{-q \tau_{b}^{+}} 1_{\left\{\tau_{b}^{+}<\tau_{0}^{-}\right\}}\right]=\frac{W^{(q)}(x)}{W^{(q)}(b)}$.

It is known (see e.g. (5) in Albrecher et al., 2016 and (8) in Landriault et al., 2014) that

$\mathbb{E}_{x}\left[e^{-q \tau_{0}^{-}} e^{\theta X\left(\tau_{0}^{-}\right)} 1_{\tau_{0}^{-}<\tau_{b}^{+}}\right]=Z^{(q)}(x, \theta)-\frac{W^{(q)}(x)}{W^{q}(b)} Z^{(q)}(b, \theta)$.

In the sequel we need also the derivative of $Z^{(q)}(x, \theta)$ with respect to $\theta$ :

$$
\begin{aligned}
\frac{d}{d \theta} Z^{(q)}(x, \theta)= & x Z^{(q)}(x, \theta)+e^{\theta x}\left(-\psi^{\prime}(\theta) \int_{0}^{x} e^{-\theta y} W^{(q)}(y) d y\right. \\
& \left.+(\psi(\theta)-q) \int_{0}^{x} y e^{-\theta y} W^{(q)}(y) d y\right) .
\end{aligned}
$$

Letting $\theta=0$ in (2.8) we obtain that

$\mathbb{E}_{x}\left[e^{-q \tau_{0}^{-}} 1_{\left\{\tau_{0}^{-}<\tau_{b}^{+}\right\}}\right]=Z^{(q)}(x)-Z^{(q)}(b) \frac{W^{(q)}(x)}{W^{(q)}(b)}$.

We shall also use the $q$-resolvent measure, which has a density $u^{(q)}(x, y)$ where

$u^{(q)}(x, y) d y=\mathbb{E}_{x} \int_{0}^{\infty} e^{-q t} 1_{X_{t} \in d y, t<\tau_{0}^{-} \wedge \tau_{b}^{+}} d t$

$=\left(\frac{W^{(q)}(x) W^{(q)}(b-y)}{W^{(q)}(b)}-W^{(q)}(x-y)\right) d y$. 


\section{The joint Laplace-Stieltjes transform of the total dividends and the time to ruin - the case $I(x)=(1-\alpha) x$}

In this section we restrict ourselves to the case that a dividend of size $I(x)=(1-\alpha) x$ is paid from each gain of size $x$ that occurs while the process is above $b$ and from each overshoot of size $x$ of a gain that brings the surplus above $b$. We devote a section to this special case because it is an important case, and because it allows us to introduce the key arguments which will, in Section 4, also enable us to handle the general $I(x)$ case.

In Section 3.1 we determine the Laplace-Stieltjes transform of the total dividend in one cycle. A cycle is the time which elapses between two successive epochs at which the process reaches $b$ from above. Section 3.2 is devoted to the derivation of the joint Laplace-Stieltjes transform of the time to ruin and the dividends until ruin. In Section 3.3 we consider the expected discounted dividends for the case $I(x)=(1-\alpha) x$. In Section 3.4 Models v2 and v3 are considered. Section 3.5 focuses on numerically obtaining the optimal barrier $b$ for some given gain amount distributions. This subsection also contains some numerical results.

\subsection{The Laplace-Stieltjes transform of the total dividends in one cycle}

In this subsection we consider model v1. Assume that $\mathcal{U}(0)=$ $b+y, y>0$. Let $\mathcal{D}_{b+y}$ be the total dividends paid until the process reaches $b$. Let $\mathcal{D}$ be the total dividends paid until the process reaches $b$ when $\mathcal{U}(0)=b+Y$.

Denote by $\mathcal{B}$ a busy period of an $M / G / 1$ queue with workload described in (2.1), but with the $i$ th gain equaling $\alpha Y_{i}$, so the service time equals $\alpha Y_{i}$.

We have the following equation for $\mathcal{L}_{\mathcal{D}_{b+y}}$ and $\mathcal{L}_{\mathcal{D}}$.

\section{Proposition 3.1.}

$\mathcal{L}_{\mathcal{D}_{b+y}}(\theta)=\exp \left(-\left\{\theta(1-\alpha)+\lambda \alpha\left(1-\mathcal{L}_{\mathcal{D}}(\theta)\right)\right\} y\right)$.

Proof. Given that $\mathcal{U}(0)=b+y$ then $(1-\alpha) y$ is paid immediately as dividend and the net surplus is $b+\alpha y$. Assume that during time $\alpha y$ there are exactly $n$ Poisson arrivals. Each one of the arrivals starts a regular busy period (which can also be infinite). Let $\mathcal{B}_{i}$ and $\mathcal{D}_{i}$ be, respectively, the $i$ th busy period and the amount of dividends paid in the $i$ th busy period. Those busy periods are independent, and distributed as the busy period in an M/G/1 queue with arrival rate $\lambda$ and service time distributed as $\alpha Y$. Thus, given that $N(\alpha y)=n \geq 0$, using a well-known branching argument that has been developed for the busy period LST in an $M / G / 1$ queue (cf. pp. 60-61 of Takács. 1962),

$\mathbb{E}\left[\exp \left(-\theta \mathcal{D}_{b+y}\right) 1_{\mathcal{D}_{b+y}<\infty} \mid N(\alpha y)=n\right]=e^{-\theta(1-\alpha) y} \mathcal{L}_{\mathcal{D}}^{n}(\theta)$.

By unconditioning,

$\mathcal{L}_{\mathcal{D}_{b+y}}(\theta)=\sum_{n=0}^{\infty} e^{-\theta(1-\alpha) y} e^{-\lambda \alpha y} \frac{(\lambda \alpha y)^{n}}{n !} \mathcal{L}_{\mathcal{D}}^{n}(\theta)$

$=\exp \left(-\left\{\theta(1-\alpha)+\lambda \alpha\left(1-\mathcal{L}_{\mathcal{D}}(\theta)\right)\right\} y\right)$.

Proposition 3.2. For $\operatorname{Re} \theta \geq 0, \mathcal{L}_{\mathcal{D}}(\theta)$ satisfies the following equation:

$\mathcal{L}_{\mathcal{D}}(\theta)=\mathcal{L}_{Y}\left(\theta(1-\alpha)+\lambda \alpha\left(1-\mathcal{L}_{\mathcal{D}}(\theta)\right)\right)$.

It is the unique solution of the equation $x=\mathcal{L}_{Y}(\theta(1-\alpha)+\lambda \alpha(1-x))$ with $|x|<1$ if $\operatorname{Re} \theta>0$ and if $\operatorname{Re} \theta \geq 0$ and $\lambda \alpha \mathbb{E}[Y]<1$, i.e., if $\Phi_{1}(0)=0$; if $\Phi_{1}(0)>0$, then $\mathcal{L}_{\mathcal{D}}(0)=1$ is the unique solution of the equation with $|x| \leq 1$.
Proof. To show that $\mathcal{L}_{\mathcal{D}}(\theta)$ satisfies (3.4), we replace $y$ by the random variable $Y$ in (3.3) and take expectations. The uniqueness follows by an application of Rouché's theorem (see, e.g., the proof of a very similar result for the LST of the busy period in the $M / G / 1$ queue on pp. 47-48 of Takács, 1962).

3.2. The joint Laplace-Stieltjes transform of the time to ruin and the dividends until ruin

Unless written otherwise we consider the model v1. Let $\mathcal{D}_{\text {tot }}^{(u)}$ be the total dividends paid until ruin when the initial surplus is $u$ and let $\mathcal{P}_{\text {tot }}^{(u)}$ be the time until ruin when the initial surplus is $u$. In this section we obtain their joint Laplace-Stieltjes transform $\mathbb{E}\left[e^{-\theta \mathcal{D}_{\text {tot }}^{(u)}-\beta \mathcal{P}_{\text {tot }}^{(u)}}\right]$, expressed in the function $Z^{(q)}(x, \theta)$ that was defined in (2.8).

Let $C_{u}$ be the time until the $\mathcal{U}$ process upcrosses $b$ given that the initial surplus is $u$, and let $R_{u}$ be the overshoot when this event occurs. Let $P_{u}$ be the period that starts when $\mathcal{U}$ overshoots $b$ and ends when it again reaches $b$, given that $\mathcal{U}(0)=u$. Let $H_{u}$ be the time the process reaches 0 starting at $u$. For future use we introduce

$\nu(\theta):=\theta(1-\alpha)+\lambda \alpha\left(1-\mathcal{L}_{\mathcal{D}}(\theta)\right)$.

\section{Proposition 3.3.}

(i) For $u \leq b$,

$$
\mathbb{E}\left[e^{-\theta \mathcal{D}_{\text {tot }}^{(u)}-\beta \mathcal{P}_{\text {tot }}^{(u)}} 1_{\left\{\mathcal{D}_{\text {tot }}^{(u)}<\infty, \mathcal{P}_{\text {tot }}^{(u)}<\infty\right\}}\right]=\frac{Z^{(\beta)}\left(b-u, v\left(\frac{\beta \alpha}{1-\alpha}+\theta\right)\right)}{Z^{(\beta)}\left(b, v\left(\frac{\beta \alpha}{1-\alpha}+\theta\right)\right)} .
$$

(ii) For $u>b$,

$$
\mathbb{E}\left[e^{-\theta \mathcal{D}_{\text {tot }}^{(u)}-\beta \mathcal{P}_{\text {tot }}^{(u)}} 1_{\left\{\mathcal{D}_{\text {tot }}^{(u)}<\infty, \mathcal{P}_{\text {tot }}^{(u)}<\infty\right\}}\right]=\frac{e^{-v\left(\frac{\beta \alpha}{1-\alpha}+\theta\right)(u-b)}}{Z^{(\beta)}\left(b, v\left(\frac{\beta \alpha}{1-\alpha}+\theta\right)\right)} .
$$

Proof.

(i) $\mathcal{U}(0)=u \leq b$.

$$
\begin{aligned}
& \mathbb{E}\left[e^{-\theta \mathcal{D}_{\text {tot }}^{(u)}-\beta \mathcal{P}_{\text {tot }}^{(u)}}\right] \\
& =\mathbb{E}\left[e^{-\beta H_{u}} 1_{\left\{H_{u}<C_{u}\right\}}\right]+\mathbb{E}\left[e^{-\beta\left(C_{u}+P_{u}\right)-\theta \mathcal{D}_{b+R u}} 1_{\left\{C_{u}<H_{u}\right\}}\right] \mathbb{E}\left[e^{-\theta \mathcal{D}_{\text {tot }}^{(b)}-\beta \mathcal{P}_{\text {tot }}^{(b)}}\right] .
\end{aligned}
$$

Notice that

$P_{u}=\frac{\alpha}{1-\alpha} \mathcal{D}_{b+R_{u}}$,

thus, by (3.3) and (3.5),

$$
\begin{aligned}
& \mathbb{E}\left[e^{-\beta\left(C_{u}+P_{u}\right)-\theta \mathcal{D}_{b+R}} 1_{\left\{C_{u}<H_{u}\right\}} \mid R_{u}=y\right] \\
& =\mathbb{E}\left[e^{-\beta C_{u}-v\left(\frac{\beta \alpha}{1-\alpha}+\theta\right) y} 1_{\left\{C_{u}<H_{u}\right\}}\right] .
\end{aligned}
$$

Hence, by (3.9) and the strong Markov property:

$$
\begin{aligned}
\mathbb{E} & {\left[e^{-\theta \mathcal{D}_{\text {tot }}^{(u)}-\beta \mathcal{P}_{\text {tot }}^{(u)}} 1_{\left\{\mathcal{D}_{\text {tot }}^{(u)}<\infty, \mathcal{P}_{\text {tot }}^{(u)}<\infty\right\}}\right] } \\
= & \mathbb{E}\left[e^{-\beta H_{u}} 1_{\left\{H_{u}<C_{u}\right\}}\right]+\mathbb{E}\left[e^{-\beta C_{u}-\left(\frac{\beta \alpha}{1-\alpha}+\theta\right) \mathcal{D}_{b+R_{u}}} 1_{\left\{C_{u}<H_{u}\right\}}\right] \\
& \times \mathbb{E}\left[e^{-\theta \mathcal{D}_{\text {tot }}^{(b)}-\beta \mathcal{P}_{\text {tot }}^{(b)}} 1_{\left\{\mathcal{D}_{\text {tot }}^{(b)}<\infty, \mathcal{P}_{\text {tot }}^{(b)}<\infty\right\}}\right] \\
= & \mathbb{E}\left[e^{-\beta H_{u}} 1_{\left\{H_{u}<C_{u}\right\}}\right]+\mathbb{E}\left[e^{-\beta C_{u}-v\left(\frac{\beta \alpha}{1-\alpha}+\theta\right) R_{u}} 1_{\left\{C_{u}<H_{u}\right\}}\right] \\
& \times \mathbb{E}\left[e^{-\theta \mathcal{D}_{\text {tot }}^{(b)}-\beta \mathcal{P}_{\text {tot }}^{(b)}} 1_{\left\{\mathcal{D}_{\text {tot }}^{(b)}<\infty, \mathcal{P}_{\text {tot }}^{(b)}<\infty\right\}}\right] .
\end{aligned}
$$

$C_{u}$ is the same as $\tau_{0}^{-}$- the time to ruin in the classical risk model with initial surplus $b-u$. Similarly, $H_{u}$ is the same as $\tau_{b}^{+}$on $\tau_{b}^{+}<\tau_{0}^{-}$-the first time to reach $b$ starting at $b-u$ in the classical risk model $X$. Thus, applying (2.11) and (2.12),

$\mathbb{E}\left[e^{-\theta \mathcal{D}_{\text {tot }}^{(u)}-\beta \mathcal{P}_{\text {tot }}^{(u)}} 1_{\left\{\mathcal{D}_{\text {tot }}^{(u)}<\infty, \mathcal{P}_{\text {tot }}^{(u)}<\infty\right\}}\right]$ 


$$
\begin{aligned}
& =\mathbb{E}_{b-u}\left[e^{-\beta \tau_{b}^{+}} 1_{\left\{\tau_{b}^{+}<\tau_{0}^{-}\right\}}\right] \\
& +\mathbb{E}_{b-u}\left[e^{-\beta \tau_{0}^{-}+v\left(\frac{\beta \alpha}{1-\alpha}+\theta\right) X\left(\tau_{0}^{-}\right)} 1_{\left\{\tau_{0}^{-}<\tau_{b}^{+}\right\}}\right] \mathbb{E}\left[e^{-\theta \mathcal{D}_{\text {tot }}^{(b)}-\beta \mathcal{P}_{\text {tot }}^{(b)}} 1_{\left\{\mathcal{D}_{\text {tot }}^{(b)}<\infty, \mathcal{P}_{\text {tot }}^{(b)}<\infty\right\}}\right] \\
& =\frac{W^{(\beta)}(b-u)}{W^{(\beta)}(b)}+\left(Z^{(\beta)}\left(b-u, v\left(\frac{\beta \alpha}{1-\alpha}+\theta\right)\right)\right. \\
& \left.-W^{(\beta)}(b-u) \frac{Z^{(\beta)}\left(b, v\left(\frac{\beta \alpha}{1-\alpha}+\theta\right)\right)}{W^{(\beta)}(b)}\right) \mathbb{E}\left[e^{-\theta \mathcal{D}_{\text {tot }}^{(b)}-\beta \mathcal{P}_{\text {tot }}^{(b)}} 1_{\left\{\mathcal{D}_{\text {tot }}^{(b)}<\infty, \mathcal{P}_{\text {tot }}^{(b)}<\infty\right\}}\right] .
\end{aligned}
$$

$\mathbb{E}\left[e^{-\theta \mathcal{D}_{\text {tot }}^{(b)}-\beta \mathcal{P}_{\text {tot }}^{(b)}} 1_{\left\{\mathcal{D}_{\text {tot }}^{(b)}<\infty, \mathcal{P}_{\text {tot }}^{(b)}<\infty\right\}}\right]$ is obtained by substituting $u=$ $b$ in (3.11):

$\mathbb{E}\left[e^{-\theta \mathcal{D}_{\text {tot }}^{(b)}-\beta \mathcal{P}_{\text {tot }}^{(b)}} 1_{\left\{\mathcal{D}_{\text {tot }}^{(b)}<\infty, \mathcal{P}_{\text {tot }}^{(b)}<\infty\right\}}\right]=\frac{1}{Z^{(\beta)}\left(b, v\left(\frac{\beta \alpha}{1-\alpha}+\theta\right)\right)}$.

Thus (3.6) follows.

(ii) $\mathcal{U}(0)=u>b$.

In this case $C_{u}=0$ and $R_{u}=u-b$. By (3.3) and (3.8),

$$
\begin{aligned}
& \mathbb{E}\left[e^{-\theta \mathcal{D}_{\text {tot }}^{(u)}-\beta \mathcal{P}_{\text {tot }}^{(u)}} 1_{\left\{\mathcal{D}_{\text {tot }}^{(u)}<\infty, \mathcal{P}_{\text {tot }}^{(u)}<\infty\right\}}\right] \\
& =e^{-v\left(\frac{\beta \alpha}{1-\alpha}+\theta\right)(u-b)} \mathbb{E}\left[e^{-\theta \mathcal{D}_{\text {tot }}^{(b)}-\beta P_{\text {tot }}^{(b)}} 1_{\left\{\mathcal{D}_{\text {tot }}^{(b)}<\infty, \mathcal{P}_{\text {tot }}^{(b)}<\infty\right\}}\right] \\
& =\frac{e^{-v\left(\frac{\beta \alpha}{1-\alpha}+\theta\right)(u-b)}}{Z^{(\beta)}\left(b, v\left(\frac{\beta \alpha}{1-\alpha}+\theta\right)\right)} \cdot
\end{aligned}
$$

Remark 3.1. The LSTs of $\mathcal{P}_{\text {tot }}^{(u)}$ and of $\mathcal{D}_{\text {tot }}^{(u)}$ immediately follow from the theorem:

For $\mathcal{U}(0)=u \leq b$,

$\mathbb{E}\left[e^{-\beta \mathcal{P}_{\text {tot }}^{(u)}} 1_{\left\{\mathcal{P}_{\text {tot }}^{(u)}<\infty\right\}}\right]=\frac{Z^{(\beta)}\left(b-u, v\left(\frac{\beta \alpha}{1-\alpha}\right)\right)}{Z^{(\beta)}\left(b, v\left(\frac{\beta \alpha}{1-\alpha}\right)\right)}$,

$\mathbb{E}\left[e^{-\theta \mathcal{D}_{\text {tot }}^{(u)}} 1_{\left\{\mathcal{D}_{\text {tot }}^{(u)}<\infty\right\}}\right]=\frac{Z^{(0)}(b-u, v(\theta))}{Z^{(0)}(b, v(\theta))}$,

and for $\mathcal{U}(0)=u>b$,

$\mathbb{E}\left[e^{-\beta \mathcal{P}_{\text {tot }}^{(u)}} 1_{\left\{\mathcal{P}_{\text {tot }}^{(u)}<\infty\right\}}\right]=\frac{\mathrm{e}^{-v\left(\frac{\beta \alpha}{1-\alpha}\right)(u-b)}}{Z^{(\beta)}\left(b, v\left(\frac{\beta \alpha}{1-\alpha}\right)\right)}$,

$\mathbb{E}\left[e^{-\theta \mathcal{D}_{\text {tot }}^{(u)}} 1_{\left\{\mathcal{D}_{\text {tot }}^{(u)}<\infty\right\}}\right]=\frac{\mathrm{e}^{-v(\theta)(u-b)}}{Z^{(0)}(b, v(\theta))}$.

Next, we obtain the ruin probability for this model. Recall that above $b$ the surplus process behaves as $\mathbb{U}$, with Laplace exponent $\psi_{1}$ with inverse $\Phi_{1}$. The Laplace-Stieltjes transform of $\mathcal{B}$ can also be written as follows:

$\mathcal{L}_{\mathcal{B}}(\theta)=\mathbb{E}\left[e^{-\Phi_{1}(\theta) \alpha Y}\right]=\mathcal{L}_{\alpha Y}\left(\Phi_{1}(\theta)\right)$.

Corollary 3.1. The ruin probability for the dual model $\mathcal{U}$ with $I(x)=$ $(1-\alpha) x$ is 1 if $\psi_{1}^{\prime}(0) \geq 0$. Otherwise it equals

$$
\begin{aligned}
& \frac{Z\left(b-u, \lambda \alpha\left(1-\mathcal{L}_{\alpha Y}\left(\Phi_{1}(0)\right)\right)\right)}{Z\left(b, \lambda \alpha\left(1-\mathcal{L}_{\alpha Y}\left(\Phi_{1}(0)\right)\right)\right)} \quad \text { if } \quad u \leq b, \\
& \frac{e^{-\lambda \alpha\left(1-\mathcal{L}_{\alpha Y}\left(\Phi_{1}(0)\right)\right)}}{Z\left(b, \lambda \alpha\left(1-\mathcal{L}_{\alpha Y}\left(\Phi_{1}(0)\right)\right)\right)} \quad \text { if } \quad u>b .
\end{aligned}
$$

Proof. Substitute $\theta=\beta=0$ in (3.6) and (3.7) and notice that

$$
\begin{aligned}
\nu(0) & =\lambda \alpha\left(1-\mathcal{L}_{\mathcal{D}}(0)\right)=\lambda \alpha\left(1-\mathcal{L}_{\mathcal{B}}(0)\right) \\
& =\lambda \alpha\left(1-\mathcal{L}_{\alpha Y}\left(\Phi_{1}(0)\right)\right) .
\end{aligned}
$$

Now observe that $\Phi_{1}(0)=0$ if $\psi_{1}^{\prime}(0) \geq 0$ (i.e., if $\lambda \alpha \mathbb{E}[Y] \leq 1$ ) and $\Phi_{1}(0)>0$ if $\psi_{1}^{\prime}(0)<0$.

To obtain the joint Laplace-Stieltjes transform of the time to ruin and the dividends paid until ruin for versions v2 and v3 we modify some of the above definitions. Let

$\tilde{v}(\theta)=\lambda\left(1-\mathcal{L}_{\mathcal{D}}(\theta)\right)$.
First consider model v2. By similar arguments as leading to Proposition 3.3 the Laplace transform of the amount of dividends until the first time the process reaches $b$ starting at $u>b$ is

$\mathcal{L}_{u}(\theta)=e^{-\tilde{v}(\theta)(u-b)}$.

Since no dividends are paid at time 0 the following relationship holds:

$P_{u}=(u-b)+\frac{\alpha}{1-\alpha} \mathcal{D}_{u}$

The proof of the following proposition is similar to the proof of Proposition 3.3:

Proposition 3.4. The joint Laplace-Stieltjes transform of the time to ruin and the dividends until ruin for the model $v 2$ is: For $\mathcal{U}(0)=u \leq b$ it is given as in (3.6) and for $\mathcal{U}(0)=u>b$ :

$\mathbb{E}\left[e^{-\theta \mathcal{D}_{\text {tot }}^{(u)}-\beta \mathcal{P}_{\text {tot }}^{(u)}} 1_{\left\{\mathcal{D}_{\text {tot }}^{(u)}<\infty, \mathcal{P}_{\text {tot }}^{(u)}<\infty\right\}}\right]=\frac{e^{-\left(\beta+\tilde{\nu}\left(\frac{\beta \alpha}{1-\alpha}+\theta\right)\right)(u-b)}}{Z^{(\beta)}\left(b, v\left(\frac{\beta \alpha}{1-\alpha}+\theta\right)\right)}$.

Now consider the joint Laplace-Stieltjes transform of the time to ruin and dividends paid until ruin for model v3. In Proposition $3.5 \mathcal{D}_{\text {tot }}^{(u)}$ and $\mathcal{P}_{\text {tot }}^{(u)}$ denote the total dividends and the time to ruin for model v3.

Proposition 3.5. For model v3:

(i) For $u \leq b$,

$$
\begin{aligned}
& \mathbb{E}\left[e^{-\theta \mathcal{D}_{\text {tot }}^{(u)}-\beta \mathcal{P}_{\text {tot }}^{(u)}} 1_{\left\{\mathcal{D}_{\text {tot }}^{(u)}<\infty, \mathcal{P}_{\text {tot }}^{(u)}<\infty\right\}}\right] \\
& =\frac{Z^{(\beta)}\left(b-u, \beta+\tilde{v}\left(\frac{\beta \alpha}{1-\alpha}+\theta\right)\right)}{Z^{(\beta)}\left(b, \beta+\tilde{v}\left(\frac{\beta \alpha}{1-\alpha}+\theta\right)\right)} .
\end{aligned}
$$

(ii) For $u>b$,

$$
\begin{aligned}
& \mathbb{E}\left[e^{-\theta \mathcal{D}_{\text {tot }}^{(u)}-\beta \mathcal{P}_{\text {tot }}^{(u)}} 1_{\left\{\mathcal{D}_{\text {tot }}^{(u)}<\infty, \mathcal{P}_{\text {tot }}^{(u)}<\infty\right\}}\right] \\
& =\frac{e^{-\left(\beta+\tilde{v}\left(\frac{\beta \alpha}{1-\alpha}+\theta\right)\right)(u-b)}}{Z^{(\beta)}\left(b, \beta+\tilde{v}\left(\frac{\beta \alpha}{1-\alpha}+\theta\right)\right)} .
\end{aligned}
$$

Proof.

(i) $\mathcal{U}(0)=u \leq b$.

$$
\begin{aligned}
& \mathbb{E}\left[e^{-\theta \mathcal{D}_{\text {tot }}^{(u)}-\beta \mathcal{P}_{\text {tot }}^{(u)}} 1_{\left\{\mathcal{D}_{\text {tot }}^{(u)}<\infty, \mathcal{P}_{\text {tot }}^{(u)}<\infty\right\}}\right] \\
& =\mathbb{E}\left[e^{-\beta H_{u}} 1_{\left\{H_{u}<C_{u}\right\}}\right]+\mathbb{E}\left[e^{-\beta\left(C_{u}+\mathcal{P}_{u}\right)-\theta \mathcal{D}_{b+R_{u}}} 1_{\left\{C_{u}<H_{u}\right\}}\right] \\
& \quad \times \mathbb{E}\left[e^{-\theta \mathcal{D}_{\text {tot }}^{(b)}-\beta \mathcal{P}_{\text {tot }}^{(b)}} 1_{\left\{\mathcal{D}_{\text {tot }}^{(b)}<\infty, \mathcal{P}_{\text {tot }}^{(b)}<\infty\right\}}\right] .
\end{aligned}
$$

By (3.16),

$P_{u}=R_{u}+\frac{\alpha}{1-\alpha} \mathcal{D}_{b+R_{u}}$.

Applying (3.15) yields

$$
\begin{gathered}
\mathbb{E}\left[e^{-\beta\left(C_{u}+P_{u}\right)-\theta \mathcal{D}_{b+R_{u}}} 1_{\left\{C_{u}<H_{u}\right\}} \mid R_{u}=y\right] \\
=\mathbb{E}\left[e^{-\beta C_{u}-\left(\beta+\tilde{\nu}\left(\frac{\beta \alpha}{1-\alpha}+\theta\right)\right) y} 1_{\left\{C_{u}<H_{u}\right\}}\right] .
\end{gathered}
$$

Hence, by (3.21) and the strong Markov property:

$$
\begin{aligned}
& \mathbb{E}\left[e^{-\theta \mathcal{D}_{\text {tot }}^{(u)}-\beta \mathcal{P}_{\text {tot }}^{(u)}} 1_{\left\{\mathcal{D}_{\text {tot }}^{(u)}<\infty, \mathcal{P}_{\text {tot }}^{(u)}<\infty\right\}}\right] \\
& =\mathbb{E}\left[e^{-\beta H_{u}} 1_{\left\{H_{u}<C_{u}\right\}}\right]+\mathbb{E}\left[e^{-\beta C_{u}-\tilde{v}\left(\frac{\beta \alpha}{1-\alpha}+\theta\right) R_{u}} 1_{\left\{C_{u}<H_{u}\right\}}\right] \\
& \quad \times \mathbb{E}\left[e^{-\theta \mathcal{D}_{\text {tot }}^{(b)}-\beta \mathcal{P}_{\text {tot }}^{(b)}} 1_{\left\{\mathcal{D}_{\text {tot }}^{(b)}<\infty, \mathcal{P}_{\text {tot }}^{(b)}<\infty\right\}}\right] .
\end{aligned}
$$


Similar arguments as those leading to (3.11) yield

$$
\begin{aligned}
\mathbb{E} & {\left[e^{-\theta \mathcal{D}_{\text {tot }}^{(u)}-\beta \mathcal{P}_{\text {tot }}^{(u)}} 1_{\left\{\mathcal{D}_{\text {tot }}^{(u)}<\infty, \mathcal{P}_{\text {tot }}^{(u)}<\infty\right\}}\right] } \\
= & \mathbb{E}_{b-u}\left[e^{-\beta \tau_{b}^{+}} 1_{\left\{\tau_{b}^{+}<\tau_{0}^{-}\right\}}\right] \\
& +\mathbb{E}_{b-u}\left[e^{-\beta \tau_{0}^{-}+\left(\beta+\tilde{v}\left(\frac{\beta \alpha}{1-\alpha}+\theta\right)\right) X\left(\tau_{0}^{-}\right)} 1_{\left\{\tau_{0}^{-}<\tau_{b}^{+}\right\}}\right] \\
& \times \mathbb{E}\left[e^{-\theta \mathcal{D}_{\text {tot }}^{(b)}-\beta \mathcal{P}_{\text {tot }}^{(b)}} 1_{\left\{\mathcal{D}_{\text {tot }}^{(u)}<\infty, \mathcal{P}_{\text {tot }}^{(u)}<\infty\right\}}\right] \\
= & \frac{W^{(\beta)}(b-u)}{W^{(\beta)}(b)}+\left[Z^{(\beta)}\left(b-u, \beta+\tilde{v}\left(\frac{\beta \alpha}{1-\alpha}+\theta\right)\right)\right. \\
& \left.-W^{(\beta)}(b-u) \frac{Z^{(\beta)}\left(b, \beta+\tilde{v}\left(\frac{\beta \alpha}{1-\alpha}+\theta\right)\right)}{W^{(\beta)}(b)}\right] \\
& \times \mathbb{E}\left[e^{-\theta \mathcal{D}_{\text {tot }}^{(b)}-\beta \mathcal{P}_{\text {tot }}^{(b)}} 1_{\left\{\mathcal{D}_{\text {tot }}^{(b)}<\infty, \mathcal{P}_{\text {tot }}^{(b)}<\infty\right\}}\right] .
\end{aligned}
$$

$\mathbb{E}\left[e^{-\theta \mathcal{D}_{\text {tot }}^{(b)}-\beta \mathcal{P}_{\text {tot }}^{(b)}} 1_{\left\{\mathcal{D}_{\text {tot }}^{(b)}<\infty, \mathcal{P}_{\text {tot }}^{(b)}<\infty\right\}}\right]$ is obtained by substituting $u=$ $b$ in (3.23):

$$
\mathbb{E}\left[e^{-\theta \mathcal{D}_{\text {tot }}^{(b)}-\beta \mathcal{P}_{\text {tot }}^{(b)}} 1_{\left\{\mathcal{D}_{\text {tot }}^{(b)}<\infty, \mathcal{P}_{\text {tot }}^{(b)}<\infty\right\}}\right]=\frac{1}{Z^{(\beta)}\left(b, \beta+\tilde{v}\left(\frac{\beta \alpha}{1-\alpha}+\theta\right)\right)} .
$$

Thus (3.17) follows.

(ii) $\mathcal{U}(0)=u>b$.

In this case $C_{u}=0$ and $R_{u}=u-b$. By (3.15) and (3.20),

$$
\begin{aligned}
& \mathbb{E}\left[e^{-\theta \mathcal{D}_{\text {tot }}^{(u)}-\beta \mathcal{P}_{\text {tot }}^{(u)}} 1_{\left\{\mathcal{D}_{\text {tot }}^{(u)}<\infty, \mathcal{P}_{\text {tot }}^{(u)}<\infty\right\}}\right] \\
& =e^{-\left(\beta+\tilde{v}\left(\frac{\beta \alpha}{1-\alpha}+\theta\right)\right)(u-b)} \mathbb{E}\left[e^{-\theta \mathcal{D}_{\text {tot }}^{(b)}-\beta P_{\text {tot }}^{(b)}} 1_{\left\{\mathcal{D}_{\text {tot }}^{(b)}<\infty, \mathcal{P}_{\text {tot }}^{(b)}<\infty\right\}}\right] \\
& =\frac{e^{-\left(\beta+\tilde{v}\left(\frac{\beta \alpha}{1-\alpha}+\theta\right)\right)(u-b)}}{Z^{(\beta)}\left(b, \beta+\tilde{v}\left(\frac{\beta \alpha}{1-\alpha}+\theta\right)\right)} \cdot
\end{aligned}
$$

\subsection{The expected discounted dividends for $I(x)=(1-\alpha) x$}

In this subsection we consider model v1; models v2 and v3 will be treated in the next subsection. Let $\mathcal{V}^{(q)}(u, b)$ be the expected discounted dividends until the process $\mathcal{U}$ reaches $b$ the first time from above.

\section{Proposition 3.6.}

(i) Let $\mathcal{U}(0)=u=b+y, y>0$. Then

$$
\mathcal{V}^{(q)}(u, b)=(1-\alpha) y+V(q) \frac{\lambda}{\eta}(1-\exp (-\alpha \eta y)),
$$

where

$$
\begin{aligned}
V(q) & =\mathbb{E}\left[\mathcal{V}^{(q)}(b+Y, b)\right] \\
& =(1-\alpha) \mathbb{E}[Y]+V(q) \frac{\lambda}{\eta}\left(1-\mathcal{L}_{Y}(\alpha \eta)\right),
\end{aligned}
$$

i.e.,

$V(q)=\frac{(1-\alpha) \mathbb{E}[Y]}{1-\frac{\lambda}{\eta}\left(1-\mathcal{L}_{Y}(\alpha \eta)\right)}$,

and

$$
\eta=q+\lambda\left(1-\mathcal{L}_{\mathcal{B}}(q)\right)
$$

(ii) Let $\mathcal{U}(0)=u \leq b$ then

$$
\begin{aligned}
& \mathcal{V}^{(q)}(u, b)=-(1-\alpha)\left[\bar{Z}^{(q)}(b-u)+\frac{(1-\lambda \mathbb{E}[Y])}{q}\left(1-Z^{(q)}(b-u)\right)\right. \\
& \left.-\frac{W^{(q)}(b-u)}{W^{(q)}(b)}\left(\bar{Z}^{(q)}(b)+\frac{(1-\lambda \mathbb{E}[Y])}{q}\left(1-Z^{(q)}(b)\right)\right)\right] \\
& +V(q) \frac{\lambda}{\eta}\left(Z^{(q)}(b-u)-\frac{W^{(q)}(b-u)}{W^{(q)}(b)} Z^{(q)}(b)\right) \\
& -V(q) \frac{\lambda}{\eta}\left(Z^{(q)}(b-u, \alpha \eta)-\frac{W^{(q)}(b-u)}{W^{(q)}(b)} Z^{(q)}(b, \alpha \eta)\right),
\end{aligned}
$$

where $\bar{Z}^{(q)}(x)=\int_{0}^{x} Z^{(q)}(y) d y$.

\section{Proof.}

(i) $\mathcal{U}(0)=u=b+y, y>0$.

First we prove (3.25). $(1-\alpha) y$ is taken from the overshoot of size $y$. If there is no gain during the period $\alpha y$ then this is the amount of dividends. Assume that there are $n$ gain arrivals during time $\alpha y$. Each such arrival starts a busy period (which might be finite or infinite) of an $\mathrm{M} / \mathrm{G} / 1$ queue with arrival rate $\lambda$ and service time distributed as $\alpha Y$ (recall that we assumed that $c=1$ ). Let $N(\alpha y)$ be the number of arrivals during $\alpha y$. The dividends in the different busy periods are i.i.d. and the expected discounted (to the beginning of the busy period) dividend in such a busy period is $V(q)$. Given that $N(\alpha y)=n$, let $T_{(i)}$ be the time of the $i$ th arrival that starts such a busy period. Thus, we have to discount the dividends in the $i$ th busy period by $e^{-q\left(T_{(i)}+\sum_{j=1}^{i-1} \mathcal{B}_{j}\right)}$. Given that $N(\alpha y)=n \geq 1$, the density function of $T_{(i)}$ is as the $i$ th order statistic from a uniform distribution on $(0, \alpha y)$. Thus:

$$
\begin{aligned}
& \mathcal{V}^{(q)}(b+y, b)=(1-\alpha) y \\
& +\sum_{n=1}^{\infty} \frac{e^{-\alpha \lambda y}(\alpha \lambda y)^{n}}{n !} n V(q) \sum_{j=1}^{n}\left(\begin{array}{c}
n-1 \\
j-1
\end{array}\right) \int_{0}^{\alpha y} e^{-q v}\left(\frac{v}{\alpha y}\right)^{j-1}\left(1-\frac{v}{\alpha y}\right)^{n-j} \mathcal{L}_{\mathcal{B}}^{j-1}(q) \frac{d v}{\alpha y} \\
& =(1-\alpha) y+V(q) \alpha \lambda y \int_{u=0}^{1}\left(\sum_{m=0}^{\infty} \frac{(\alpha \lambda y)^{m} e^{-\alpha \lambda y}}{m !}\left(u \mathcal{L}_{\mathcal{B}}(q)+1-u\right)^{m} e^{-q u \alpha y}\right) d u \\
& =(1-\alpha) y+V(q) \alpha \lambda y \int_{0}^{1} \exp \left(-u \alpha y q+\alpha \lambda y\left(u \mathcal{L}_{\mathcal{B}}(q)-u\right)\right) d u \\
& =(1-\alpha) y+V(q) \frac{\lambda}{\eta}(1-\exp (-\alpha \eta y)) .
\end{aligned}
$$

(3.26) is obtained by replacing $y$ with $Y$ and taking expectation.

(ii) $\mathcal{U}(0)=u \leq b$.

Until the process $\mathcal{U}$ reaches $b$ it behaves as $U$. In terms of the process $X=b-U$, the overshoot above $b$ is the same as $-X\left(\tau_{0}^{-}\right)$, i.e., the absolute value of the deficit in the process $X$. Thus, using (3.25), the expected discounted dividends until $\mathcal{U}$ reaches $b$ (or $X$ reaches 0$), \mathcal{V}^{(q)}(u, b)$ is

$$
\mathbb{E}_{b-u}\left[e^{-q \tau_{0}^{-}} 1_{\left\{\tau_{0}^{-}<\tau_{b}^{+}\right\}}\left(-(1-\alpha) X\left(\tau_{0}^{-}\right)+V(q) \frac{\lambda}{\eta}\left(1-\exp \left(\alpha \eta X\left(\tau_{0}^{-}\right)\right)\right)\right)\right]
$$

By (2.12),

$$
\begin{aligned}
& \mathbb{E}_{b-u}\left[e^{-q \tau_{0}^{-}} e^{\alpha \eta X\left(\tau_{0}^{-}\right)} 1_{\left\{\tau_{0}^{-}<\tau_{b}^{+}\right\}}\right] \\
& =Z^{(q)}(b-u, \alpha \eta)-\frac{W^{(q)}(b-u)}{W^{q}(b)} Z^{(q)}(b, \alpha \eta) .
\end{aligned}
$$

By (2.14),

$\mathbb{E}_{b-u}\left[e^{-q \tau_{0}^{-}} 1_{\left\{\tau_{0}^{-}<\tau_{b}^{+}\right\}}\right]=Z^{(q)}(b-u)-\frac{W^{(q)}(b-u)}{W^{(q)}(b)} Z^{(q)}(b)$. 
By (2.9), (2.12), (2.13) and substituting $\psi^{\prime}(0)=1-\lambda \mathbb{E}[Y]$ (since $c=1)$ :

$$
\begin{aligned}
& \mathbb{E}_{b-u}\left[e^{-q \tau_{0}^{-}} X\left(\tau_{0}^{-}\right) 1_{\tau_{0}^{-}<\tau_{b}^{+}}\right] \\
& =\left.\frac{\partial}{\partial \theta}\left(Z^{(q)}(b-u, \theta)-\frac{W^{(q)}(b-u)}{W^{q}(b)} Z^{(q)}(b, \theta)\right)\right|_{\theta=0} \\
& =(b-u) Z^{(q)}(b-u)-(1-\lambda \mathbb{E}[Y]) \bar{W}^{(q)}(b-u)-q \int_{0}^{b-u} y W^{(q)}(y) d y \\
& -\frac{W^{(q)}(b-u)}{W^{(q)}(b)}\left(b Z^{(q)}(b)-(1-\lambda \mathbb{E}[Y]) \bar{W}^{(q)}(b)-q \int_{0}^{b} y W^{(q)}(y) d y\right),
\end{aligned}
$$

where $\bar{W}^{(q)}(x)=\int_{0}^{x} W^{(q)}(y) d y$. Notice that

$\bar{Z}^{(q)}(x)=x Z^{(q)}(x)-q \int_{0}^{x} y W^{(q)}(y) d y$.

Substituting (3.35) in (3.34) and applying (3.32)-(3.34) yields (3.29).

Substituting $u=b$ and $Z^{(q)}(0, \theta)=1$ in (3.29) we obtain that

$$
\begin{aligned}
& \mathcal{V}^{(q)}(b, b) \\
& =(1-\alpha)\left[\frac{W^{(q)}(0)}{W^{(q)}(b)}\left(b Z^{(q)}(b)-(1-\lambda \mathbb{E}[Y]) \bar{W}^{(q)}(b)-q \int_{0}^{b} y W^{(q)}(y) d y\right)\right] \\
& +V(q) \frac{\lambda}{\eta}\left(1-\frac{W^{(q)}(0)}{W^{(q)}(b)} Z^{(q)}(b)\right)-V(q) \frac{\lambda}{\eta}\left(1-\frac{W^{(q)}(0)}{W^{(q)}(b)} Z^{(q)}(b, \alpha \eta)\right) .
\end{aligned}
$$

Next we derive $\mathcal{V}_{\text {tot }}^{(q)}(u)$ - the total discounted dividends given that the initial surplus is $u$.

\section{Proposition 3.7.}

$V_{\text {tot }}^{(q)}(u)=\mathcal{V}^{(q)}(u, b)+\frac{\delta_{u} \mathcal{V}^{(q)}(b, b)}{1-\delta_{b}}$,

where for $u>b$,

$\delta_{u}=e^{-\Phi_{1}(q) \alpha(u-b)}$

and for $u \leq b$,

$\delta_{u}=Z^{(q)}\left(b-u, \alpha \Phi_{1}(q)\right)-\frac{W^{(q)}(\alpha(b-u))}{W^{(q)}(b)} Z^{(q)}\left(b, \alpha \Phi_{1}(q)\right)$.

Proof. $\delta_{u}$ is the discounted time until the process $\mathcal{U}$ reaches $b$ from above. When $u>b, \mathcal{U}$ behaves as $\mathbb{U}$. Since $(1-\alpha)(u-b)$ is paid as dividends, and the time to reach $b$ is distributed as the time that $X_{1}=\alpha(u-b)-\mathbb{U}$ reaches $u-b$ starting at 0. Thus, (3.38) follows from (2.10). For $u \leq b$ the time until $\mathcal{U}$ reaches $b$ is $C_{u}+P_{u} . C_{u}$ is the same as the time to ruin $\tau_{0}^{-}$for the risk process $X=b-U . P_{u}$ is the same as the time to reach $-X\left(\tau_{0}^{-}\right)$by the process $X_{1}$ which behaves as $-\mathbb{U}$ starting at $0 .(2.12)$ and (2.10) yield that

$$
\begin{aligned}
& \delta_{u}=\mathbb{E}\left[e^{-q\left(C_{u}+P_{u}\right)} 1_{C_{u}<H_{u}}\right]=\mathbb{E}_{b-u}\left[e^{-q \tau_{0}^{-}} e^{\Phi_{1}(q) \alpha X\left(\tau_{0}^{-}\right)}\right] \\
& =Z^{(q)}\left(b-u, \alpha \Phi_{1}(q)\right)-\frac{W^{(q)}(b-u)}{W^{(q)}(b)} Z^{(q)}\left(b, \alpha \Phi_{1}(q)\right) .
\end{aligned}
$$

Substituting $u=b$ and $W^{(q)}(0)=1$ (see Lemma 8.6 in Kyprianou, 2006) yields that

$$
\begin{aligned}
& \delta_{b}=\mathbb{E}\left[e^{-q\left(C_{b, j}+P_{b, j}\right)}\right]=\mathbb{E}_{0}\left[e^{-q \tau_{0}^{-}} e^{\Phi_{1}(q) \alpha X\left(\tau_{0}^{-}\right)}\right] \\
& =1-\frac{1}{W^{(q)}(b)} Z^{(q)}\left(b, \alpha \Phi_{1}(q)\right) .
\end{aligned}
$$

$\mathcal{V}_{\text {tot }}(u)$, the expected total discounted dividends until ruin, is given by

$$
\begin{aligned}
& \mathcal{V}_{\text {tot }}^{(q)}(u)=\mathcal{V}^{(q)}(u, b)+\delta_{u} V^{(q)}(b, b) \sum_{n=0}^{\infty} \delta_{b}^{n} \\
& =\mathcal{V}^{(q)}(u, b)+\frac{\delta_{u} \mathcal{V}^{(q)}(b, b)}{1-\delta_{b}} \cdot
\end{aligned}
$$

\subsection{The models $v 2$ and $v 3$}

First consider model v2. This model differs from model v1 only when $\mathcal{U}(0)=u>b$ since no dividends are paid at time 0 . Therefore, the total discounted dividends paid until the process reaches $b$ the first time, $\mathcal{V}_{2}^{(q)}(u, b)$ equals

$\mathcal{V}_{2}^{(q)}(u, b)=V(q) \frac{\lambda}{\eta}(1-\exp (-\eta(u-b))), \quad u>b$.

(3.42) follows from (3.30) by deleting the term $(1-\alpha) y$ and then replacing $\alpha y$ by $y$ since no dividends are paid at time 0 . The discount time until the process reaches $b$ for the first time is

$\delta_{u}^{(2)}=e^{-\Phi_{1}(q)(u-b)}$.

Thus the expected discounted dividends paid in this case for $u>b$ is as in (3.37) with the following modifications: For $u>b$, $\mathcal{V}^{(q)}(u, b)$ is replaced by $\mathcal{V}_{2}^{(q)}(u, b)$ and $\delta_{u}$ is replaced by $\delta_{u}^{(2)}$.

Now consider model v3. Recall that in this model $(1-\alpha)$ of each gain that arrives while the surplus is above $b$ is paid as dividends. Let $\mathcal{V}_{3}^{(q)}(u, b)$ be the total dividends paid until the first time the process reaches $b$ starting at $u$.

\section{Proposition 3.8.}

(i) Let $\mathcal{U}(0)=u=b+y, y>0$. Then

$$
\mathcal{V}_{3}^{(q)}(u, b)=\mathcal{V}_{2}^{(q)}(u, b)
$$

(ii) Let $\mathcal{U}(0)=u \leq b$, then

$$
\begin{aligned}
& \mathcal{V}_{3}^{(q)}(u, b)=V(q) \frac{\lambda}{\eta}\left(Z^{(q)}(b-u)-\frac{W^{(q)}(b-u)}{W^{(q)}(b)} Z^{(q)}(b)\right) \\
& -V(q) \frac{\lambda}{\eta}\left(Z^{(q)}(b-u, \eta)-\frac{W^{(q)}(b-u)}{W^{(q)}(b)} Z^{(q)}(b, \eta)\right) .
\end{aligned}
$$

\section{Proof.}

(i) $\mathcal{U}(0)=u=b+y, y>0$. (3.44) is straightforward from the discussion leading to (3.42).

(ii) $\mathcal{U}(0)=u \leq b$. Since in model $\mathrm{v} 3$ no dividends are paid when the process overshoots $b,(3.31)$ is reduced to

$$
\mathbb{E}_{b-u}\left[e^{-q \tau_{0}^{-}} 1_{\left\{\tau_{0}^{-}<\tau_{b}^{+}\right\}}\left(V(q) \frac{\lambda}{\eta}\left(1-\exp \left(\eta X\left(\tau_{0}^{-}\right)\right)\right)\right)\right] .
$$

The same arguments leading to (3.29) lead to (3.45).

\subsection{The optimal $b$}

In this subsection we want to determine the value of $b$ that maximizes the expected discounted dividend. In order to obtain the optimal barrier $b$ we consider numerical examples with $c=$ $1, \lambda=1.25$ and three gain amount distributions, all with mean 1 : 


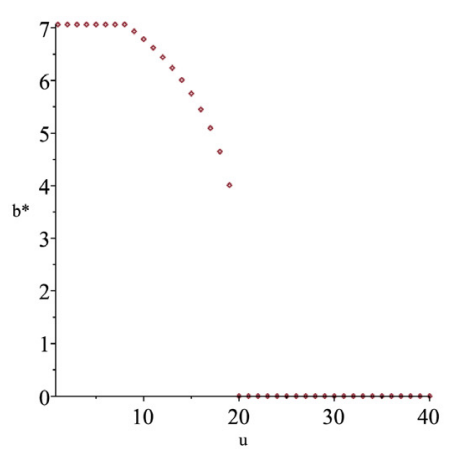

(a) $q=0.01$

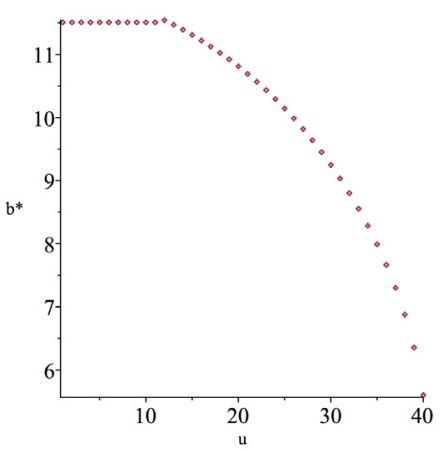

(b) $q=0.005$

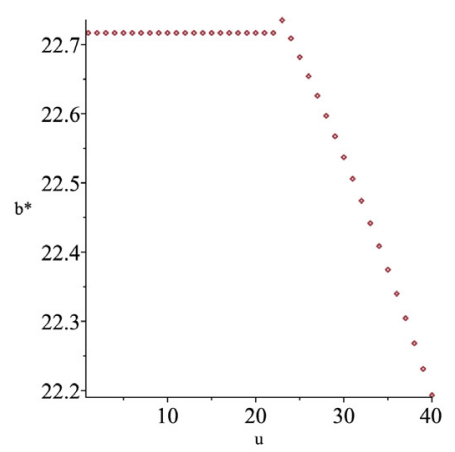

(c) $q=0.001$

Fig. 1. The optimal barrier $b^{*}$ versus initial surplus $u$ for model v1 - Exponential gains.

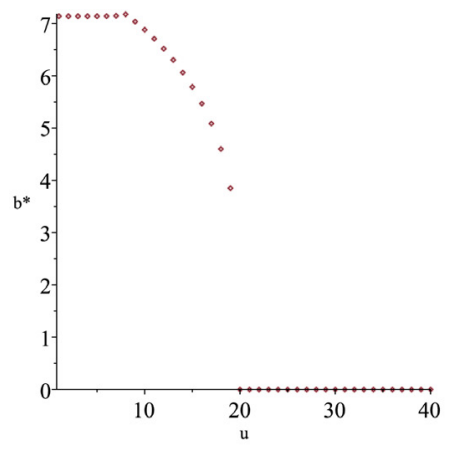

(a) $q=0.01$

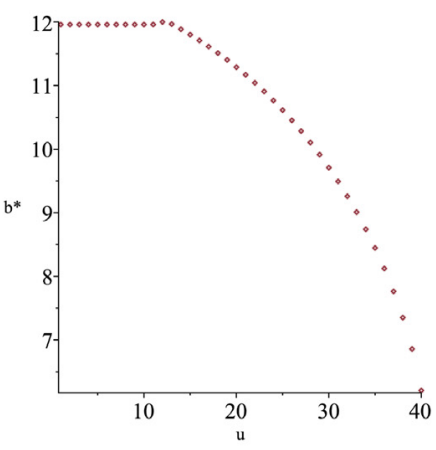

(b) $q=0.005$

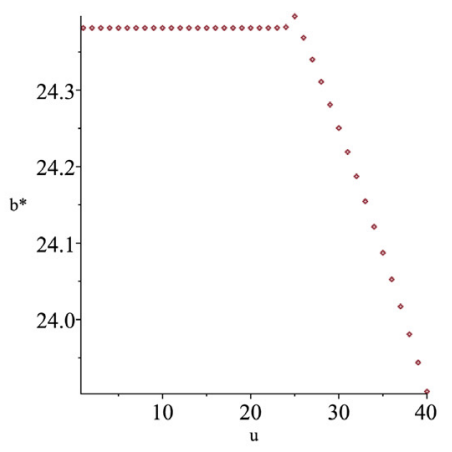

(c) $q=0.001$

Fig. 2. The optimal barrier $b^{*}$ versus initial surplus $u$ for model $v 1$ - Hyper-Exponential gains.

- Exponential with density $f(x)=e^{-x}$. In this case the scale function is

$$
W^{(q)}(x)=\frac{e^{\Phi(q) x}}{\psi^{\prime}(\Phi(q))}+\frac{e^{\xi_{1} x}}{\psi^{\prime}\left(\xi_{1}\right)},
$$

where $\Phi(q)$ and $\xi_{1}$ are the positive and negative roots of the equation

$\psi(\theta)=\theta-\lambda+\lambda \frac{1}{1+\theta}=q$.

- Hyper-exponential with density $f(x)=\frac{1}{3} 2 e^{-2 x}+\frac{2}{3} 0.8 e^{-0.8 x}$. In this case the scale function is

$W^{(q)}(x)=\frac{e^{\Phi(q) x}}{\psi^{\prime}(\Phi(q))}+\frac{e^{\xi_{1} x}}{\psi^{\prime}\left(\xi_{1}\right)}+\frac{e^{\xi_{2} x}}{\psi^{\prime}\left(\xi_{2}\right)}$,

where $\Phi(q)$ is the positive root and $\xi_{1}, \xi_{2}$ are the two negative roots of the equation

$\psi(\theta)=\theta-\lambda+\lambda\left(\frac{1}{3} \frac{2}{2+\theta}+\frac{2}{3} \frac{0.8}{0.8+\theta}\right)=q$.

- Erlang with density $f(x)=4 x e^{-2 x}$. Here the scale function is as in (3.48) where $\Phi(q)$ and $\xi_{1}, \xi_{2}$ are the positive and the two negative roots of the equation:

$\psi(\theta)=\theta-\lambda+\lambda\left(\frac{2}{2+\theta}\right)^{2}=q$.

For the derivation of the above scale functions see Egami and Yamazaki (2014). The optimal level $b^{*}$ was calculated for the three models. For each model the optimal level $b *$ was obtained for the three gain distributions and three discount factors: 0.01, 0.005 and 0.001 .

The findings demonstrate that in model $\mathrm{v} 1$ the optimal $b^{*}$ depends on the initial value $u$ as seen in Figs. 1-3. A similar phenomenon where the optimal barrier depends on the initial surplus was observed in the numerical section of Cheung and Wong (2017) and Cheung and Zhang (2018).

The common behavior of the optimal barrier for the model v1 is as follows: There is a $\tilde{b}$ where for $u<\tilde{b}$ the value of the optimal barrier $b^{*}$ does not change much with $u$. However, for $u>\tilde{b}$ the optimal value $b^{*}$ decreases rapidly with $u$. This is due to the fact that when $u>b,(1-\alpha)(u-b)$ is paid immediately, at time 0 as dividends.

The variation of the optimal barrier level as a function of the initial surplus for model v2 is less than in model 1 . When the gains are Exponentially distributed the optimal barrier is independent of the initial surplus as seen in Fig. 4. In the case where the gains have Hyper-Exponential distribution there is a very small increase of the optimal barrier in the initial level $u$, up to level $\tilde{b}$. Then for $u>\tilde{b}$ the optimal barrier $b^{*}=\tilde{b}$ as seen in Fig. 5 . In the case of Erlang gains the optimal barrier decreases a little up to level $\tilde{b}$. Then for $u>\tilde{b}$ the optimal barrier is $b^{*}=\tilde{b}$ (see Fig. 6 ).

For the model v3 the optimal dividend barrier $b^{*}$ is independent of the initial surplus $u$. The following table presents the optimal barrier $b^{*}$ for the three gains distributions and the three discount factors.

\begin{tabular}{llll}
\hline & $\mathrm{q}$ & & \\
\cline { 2 - 4 } Gain distribution & 0.01 & 0.005 & 0.001 \\
\hline Exponential & 6.1716440 & 10.6165267 & 21.8242898 \\
Hyper-Exponential & 6.1570978 & 10.9733702 & 23.3931016 \\
Erlang & 6.0153405 & 9.5898275 & 18.1925216 \\
\hline
\end{tabular}

\section{General dividends}

In this section we generalize the previous results, assuming that from each gain of size $x$ which arrives when the surplus is above $b$ 


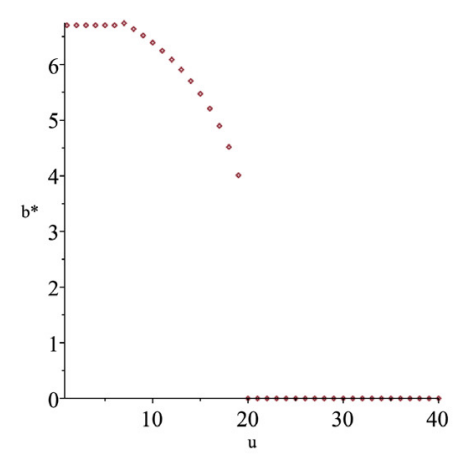

(a) $q=0.01$

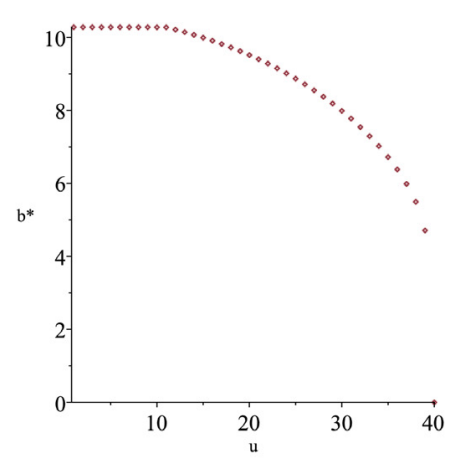

(b) $q=0.005$

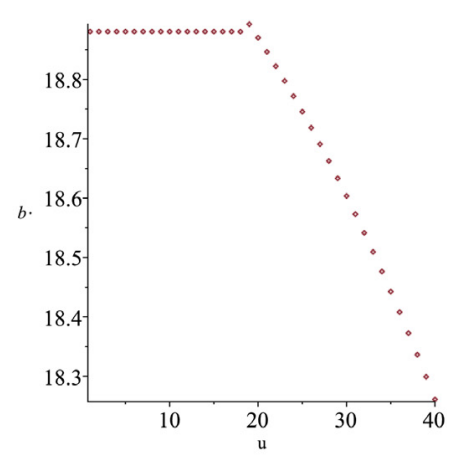

(c) $q=0.001$

Fig. 3. The optimal barrier $b^{*}$ versus initial surplus $u$ for model v1 - Erlang gains.

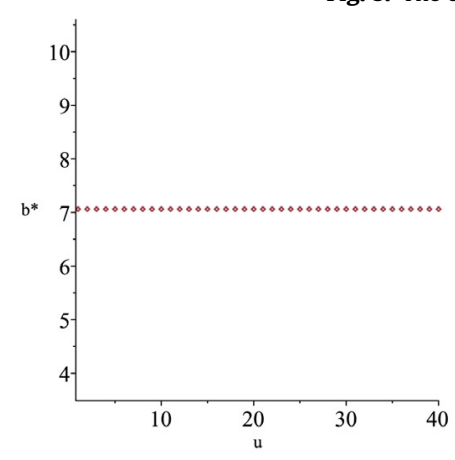

(a) $q=0.01$

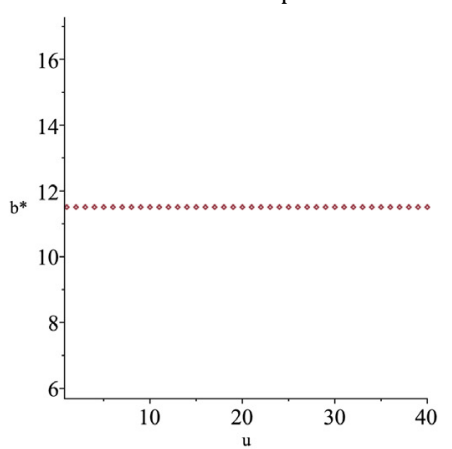

(b) $q=0.005$

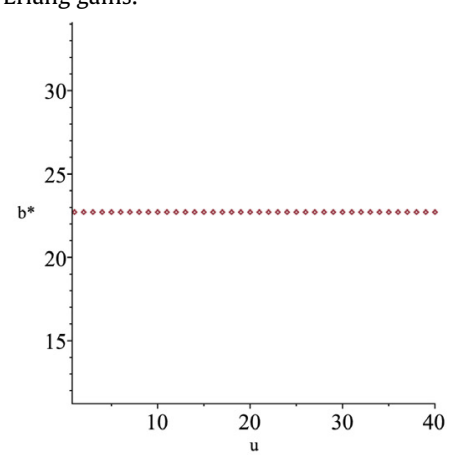

(c) $q=0.001$

Fig. 4. The optimal barrier $b^{*}$ versus initial surplus $u$ for model v2 - Exponential gains.

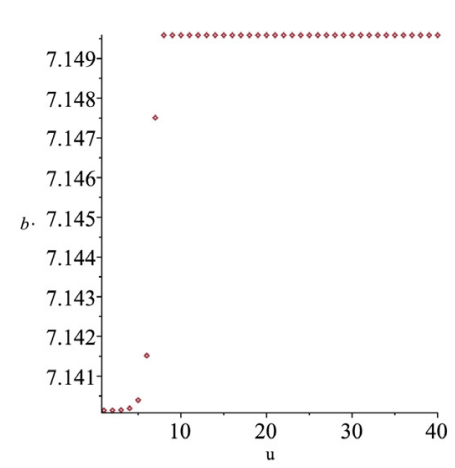

(a) $q=0.01$

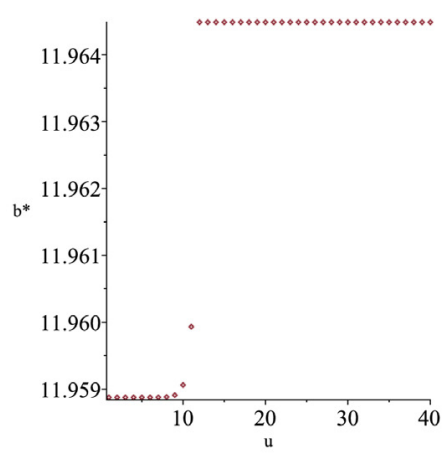

(b) $q=0.005$

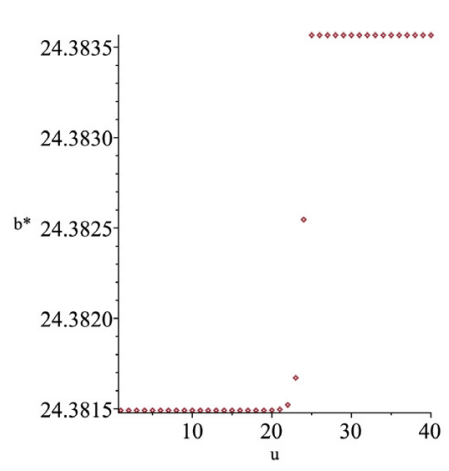

(c) $q=0.001$

Fig. 5. The optimal barrier $b^{*}$ versus initial surplus $u$ for model v2 - Hyper-Exponential gains.

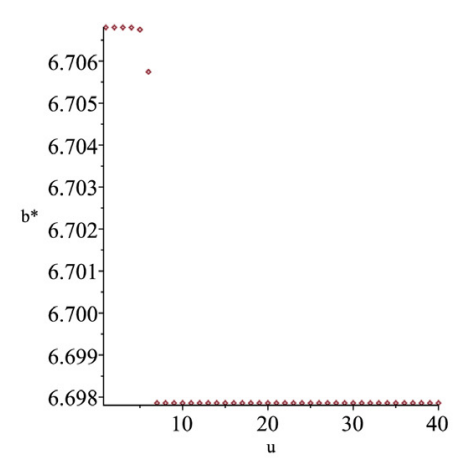

(a) $q=0.01$

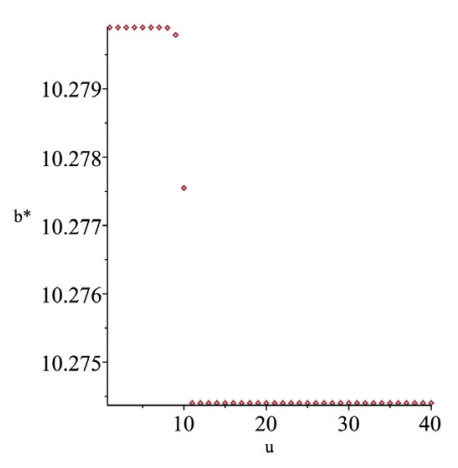

(b) $q=0.005$

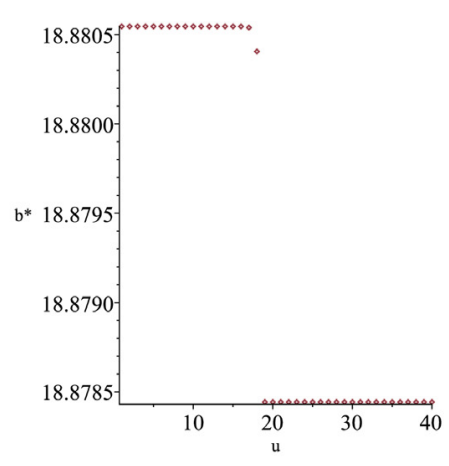

(c) $q=0.001$

Fig. 6. The optimal barrier $b^{*}$ versus initial surplus $u$ for model v2 - Erlang gains. 
or from each overshoot of size $x$ of a gain that brings the surplus above $b$, the dividend paid is $I(x)$, where $0<I(x)<x$. We restrict ourselves to model v1; results for models v2 and v3 can be obtained in a very similar way.

4.1. The Laplace-Stieltjes transform of the time to ruin and the total dividends paid

Assume $\mathcal{U}(0)=u+y$ (or that the overshoot is $y$ ). Let $\mathcal{B}_{b+y}, \mathcal{D}_{b+y}$ be, respectively, the time to reach $b$ from above and the total dividends paid until this time, given that before paying dividends $\mathcal{U}(0)=b+y \cdot \mathcal{B}$ and $\mathcal{D}$ are similarly defined with $y$ replaced by $Y$. Notice that $\mathcal{B}$ is a busy period in an $M / G / 1$ queue with Poisson arrivals at rate $\lambda$ and service distributed as $Y-I(Y)$, and $\mathcal{D}$ is the dividends paid during this period. Let

$\mathcal{L}_{\mathcal{B}_{b+y}, \mathcal{D}_{b+y}}(\beta, \theta)=\mathbb{E}\left[e^{-\beta \mathcal{B}_{b+y}-\theta \mathcal{D}_{b+y}} 1_{\left\{\mathcal{B}_{b+y}<\infty, \mathcal{D}_{b+y}<\infty\right\}}\right]$

be the joint Laplace-Stieltjes transform of $\mathcal{B}_{b+y}$ and $\mathcal{D}_{b+y}$. Then

Proposition 4.1. For $\operatorname{Re} \beta, \theta \geq 0$, the joint LST of $\mathcal{B}$ and $\mathcal{D}$ satisfies the following equation:

$\mathcal{L}_{\mathcal{B}, \mathcal{D}}(\beta, \theta)=\mathbb{E}\left[e^{-\left(\beta+\lambda-\lambda \mathcal{L}_{\mathcal{B}, \mathcal{D}}(\beta, \theta)\right) Y-\left(\theta-\beta-\lambda+\lambda \mathcal{L}_{\mathcal{B}, \mathcal{D}}(\beta, \theta)\right) I(Y)}\right]$.

It is the unique solution of the equation $x=\mathbb{E}\left[e^{-\theta I(Y)-(\beta+\lambda(1-x))(Y-I(Y))}\right]$ with $|x|<1$ if $\operatorname{Re} \beta>0, \operatorname{Re} \theta>0$ and if $\operatorname{Re} \beta \geq 0, \operatorname{Re} \theta \geq 0$ and $\lambda \mathbb{E}(Y-I(Y))<1$, i.e., if $\Phi_{1}(0)=0$; if $\Phi_{1}(0)>0$, then $\mathcal{L}_{\mathcal{B}, \mathcal{D}}(0)=1$ is the unique solution of the equation with $|x| \leq 1$.

Proof. First assume that the overshoot over $b$ is $y$. In this case the amount of dividends withdrawn immediately is $I(y)$, and the net jump is $y-I(y)$. The time until the process reaches $b$ is $y-$ $I(y)+\sum_{i=0}^{N(y-I(y))} \mathcal{B}_{i}$, where $\mathcal{B}_{i}$ are i.i.d. distributed as $\mathcal{B}$. Similarly, the amount of dividends paid until the process reaches $b$ when the overshoot is $y$ is $I(y)+\sum_{i=0}^{N(y-I(y))} \mathcal{D}_{i}$, where $\mathcal{D}_{i}$ are i.i.d. distributed as $\mathcal{D}$. Thus the joint Laplace-Stieltjes transform of the time until the process hits $b$ and the dividends paid up to this time given that the overshoot is $y, \mathcal{L}_{\mathcal{B}_{b+y}, \mathcal{D}_{b+y}}(\beta, \theta)$, is

$$
\begin{aligned}
& \mathcal{L}_{\mathcal{B}_{b+y}, \mathcal{D}_{b+y}}(\beta, \theta)=\mathbb{E}\left[e^{-\beta(y-I(y))-\theta I(y)-\sum_{j=0}^{N(y-I(y))}\left(\beta \mathcal{B}_{i}+\theta \mathcal{D}_{i}\right)}\right] \\
& =e^{-\beta(y-I(y))-\theta I(y)} \sum_{j=0}^{\infty} e^{-\lambda(y-I(y))} \frac{(\lambda(y-I(y)))^{j}}{j !}\left(\mathcal{L}_{\mathcal{B}, \mathcal{D}}(\beta, \theta)\right)^{j} \\
& =e^{-\beta(y-I(y))-\theta I(y)-\lambda(y-I(y))\left(1-\mathcal{L}_{\mathcal{B}, \mathcal{D}(\beta, \theta))}\right.}
\end{aligned}
$$

Assuming now that the overshoot is a random variable $Y$ and replacing $y$ by $Y$ in (4.2), yields (4.1). The uniqueness follows again by Rouché's theorem, cf. the proof of Proposition 3.2.

Let $\mathcal{U}(0)=u, 0 \leq u \leq b$, thus $X(0)=b-u$. First we consider the joint Laplace-Stieltjes transform of the time until the process reaches $b$ when it upcrosses $b$ before ruin, and the total dividends paid until this time.

Theorem 4.1. Let $\mathcal{U}(0)=u \leq b$. Then

$\mathbb{E}\left[e^{-\beta\left(C_{u}+P_{u}\right)} 1_{C_{u}<H_{u}} \mathcal{L}_{B_{\mathcal{U}\left(C_{u}\right)}, \mathcal{D}_{\mathcal{U}\left(C_{u}\right)}}(\beta, \theta)\right]$

$=\int_{0}^{b} \int_{z=y}^{\infty} u^{(\beta)}(b-u, y) \lambda d F_{Y}(z) \mathcal{L}_{\mathcal{B}_{b+z-y}, \mathcal{D}_{b+z-y}}(\beta, \theta) d y$.

Proof. $X(t)=b-\mathcal{U}(t)$ is a spectrally negative Lévy process and for $\mathcal{U}(0)=u, C_{u}$ and $H_{u}$ are the same as the first time that $X=b-\mathcal{U}$ downcrosses 0 or hits $b$, respectively. We apply the definition of the density $u^{(\beta)}(\cdot, \cdot)$ of the $\beta$-resolvent measure given in (2.15). $u^{(\beta)}(b-u, y) \lambda d F_{y}(z)$ is the Laplace transform of the time until the process $X$ (starting at $b-u$ ) reaches $y$ before hitting $b$ or downcrossing 0 , and then downcrosses 0 due to a claim of size $z>y$. It is the same as the Laplace transform of the time until $U$ (starting at $u$ ) reaches $b-y$ before hitting 0 or before upcrossing $b$, and then upcrosses $b$ due to a gain of size $z>y$. Multiplying it by $\mathcal{L}_{\mathcal{B}_{b+z-y}, \mathcal{D}_{b+z-y}}(\beta, \theta)$ yields the result.

To obtain the joint Laplace-Stieltjes transform of $\mathcal{D}_{\text {tot }}^{u}$ and $\mathcal{P}_{\text {tot }}^{(u)}$ we write similar equations as (3.20) and (3.23).

\section{Proposition 4.2.}

(i) Let $u \leq b$ then

$$
\begin{aligned}
& \mathbb{E}\left[e^{-\theta \mathcal{D}_{\text {tot }}^{(u)}-\beta \mathcal{P}_{\text {tot }}^{(u)}} 1_{\left\{\mathcal{D}_{\text {tot }}^{(u)}<\infty, \mathcal{P}_{\text {tot }}^{(u)}<\infty\right\}}\right]=\frac{W^{(\beta)}(b-u)}{W^{(\beta)}(b)} \\
& +\int_{0}^{b} \int_{z=y}^{\infty} u^{(\beta)}(b-u, y) \lambda d F_{Y}(z) \mathcal{L}_{\mathcal{B}_{b+z-y}, \mathcal{D}_{b+z-y}}(\beta, \theta) d y \\
& \times \mathbb{E}\left[e^{-\theta \mathcal{D}_{\text {tot }}^{(b)}-\beta \mathcal{P}_{\text {tot }}^{(b)}} 1_{\left\{\mathcal{D}_{\text {tot }}^{(b)}<\infty, \mathcal{P}_{\text {tot }}^{(b)}<\infty\right\}}\right],
\end{aligned}
$$

where $\mathbb{E}\left[e^{-\theta \mathcal{D}_{\text {tot }}^{(b)}-\beta \mathcal{P}_{\text {tot }}^{(b)}} 1_{\left\{\mathcal{D}_{\text {tot }}^{(b)}<\infty, \mathcal{P}_{\text {tot }}^{(b)}<\infty\right\}}\right]$ is obtained by substituting $u=b$ in (4.5).

(ii) Let $u=b+y, y>0$. Then

$$
\begin{aligned}
& \mathbb{E}\left[e^{-\theta \mathcal{D}_{\text {tot }}^{(u)}-\beta \mathcal{P}_{\text {tot }}^{(u)}} 1_{\left\{\mathcal{D}_{\text {tot }}^{(u)}<\infty, \mathcal{P}_{\text {tot }}^{(u)}<\infty\right\}}\right] \\
& =\mathcal{L}_{\mathcal{B}_{u}, \mathcal{D}_{u}}(\beta, \theta) \mathbb{E}\left[e^{-\theta \mathcal{D}_{\text {tot }}^{(b)}-\beta \mathcal{P}_{\text {tot }}^{(b)}} 1_{\left\{\mathcal{D}_{\text {tot }}^{(b)}<\infty, \mathcal{P}_{\text {tot }}^{(b)}<\infty\right\}}\right] .
\end{aligned}
$$

\section{Proof.}

(i) Similar arguments as in the proof of Proposition 3.3 yield that the Laplace-Stieltjes transform of the time until $\mathcal{U}$ reaches 0 before hitting $b$ (and thus ruin occurs before dividends are paid) is $\frac{W^{(\beta)}(b-u)}{W^{(\beta)}(b)}$. According to the definition (2.15), $u^{(\beta)}(b-$ $u, y) \lambda d F_{Y}(z) d y$ is the Laplace-Stieltjes transform of the time until the process equals $b-y$, and a claim of size $z>y$ occurs. Multiplying it by $\mathcal{L}_{\mathcal{B}_{b+z-y}, \mathcal{D}_{b+z-y}}(\beta, \theta)$ gives the joint LaplaceStieltjes transform of the time until the process reaches $b$ from above and the dividends paid until this time. Due to the strong Markov property, multiplying it by the joint Laplace-Stieltjes transform of the time to ruin and the dividends paid until ruin, starting at $b$, yields (4.5).

(ii) $\mathcal{L}_{\mathcal{B}_{u}, \mathcal{D}_{u}}(\beta, \theta)$ is the joint Laplace transform of the time until reaching $b$ from above and the total dividends paid up to this time. Thus, (4.5) holds due to the strong Markov property.

Below we find the ruin probability for this case.

Corollary 4.1. The ruin probability for the dual model $\mathcal{U}$ with general $I(x)$ is 1 if $\psi_{1}^{\prime}(0) \geq 0$, i.e., if $\lambda \mathbb{E}(Y-I(Y)) \leq 1$. Otherwise it equals

$\frac{W^{(0)}(b-u)}{W^{(0)}(b)}+$

$\frac{\frac{1}{W^{(0)}(b)} \int_{0}^{b} \int_{z=y}^{\infty} u^{(0)}(b-u, y) \lambda d F_{Y}(z) e^{-\lambda(z-y-I(z-y))\left(1-\mathcal{L}_{Y-I(Y)}\left(\Phi_{1}(0)\right)\right)} d y}{1-\int_{0}^{b} \int_{z=y}^{\infty} u^{(0)}(0, y) \lambda d F_{Y}(z) e^{-\lambda(z-y-I(z-y))\left(1-\mathcal{L}_{Y-I(Y)}\left(\Phi_{1}(0)\right)\right)} d y}$

if $u \leq b$ and

$\frac{\frac{1}{W^{(0)}(b)} e^{-\lambda(u-b-I(u-b))\left(1-\mathcal{L}_{Y-I(Y)}\left(\Phi_{1}(0)\right)\right)} d y}{1-\int_{0}^{b} \int_{z=y}^{\infty} u^{(0)}(0, y) \lambda d F_{Y}(z) e^{-\lambda(z-y-I(z-y))\left(1-\mathcal{L}_{Y-I(Y)}\left(\Phi_{1}(0)\right)\right)} d y}$

if $u>b$.

Proof. Solving (4.1) for $\mathcal{L}_{\mathcal{B}, \mathcal{D}}(0,0)$ yields that

$\mathcal{L}_{\mathcal{B}, \mathcal{D}}(0,0)=\mathcal{L}_{Y-I(Y)}\left(\Phi_{1}(0)\right)$. 
As seen in Proposition 4.1, $\mathcal{L}_{\mathcal{B}, \mathcal{D}}(0,0)=1$ when $\psi_{1}^{\prime}(0) \leq 0$ and $\Phi_{1}(0)=0$. In this case $\mathcal{L}_{\mathcal{B}_{b+y}, \mathcal{D}_{b+y}}(0,0)=1$. Notice that,

$\int_{0}^{b} \int_{z=y}^{\infty} u^{(0)}(b-u, y) \lambda d F_{Y}(z) d y$

$=Z^{(0)}(b-u)-\frac{Z^{(0)}(b)}{W^{(0)}(b)} W^{(0)}(b-u)=1-\frac{W^{(0)}(b-u)}{W^{(0)}(b)}$.

The first line in (4.9) is the probability that $X_{1}$ downcrosses 0 before reaching $b$ starting at $b-u$. Due to (2.14) the first equality holds. The last equality holds since $Z^{(0)}=1$.

Substituting $u=b$ and solving (4.5) for the ruin probability starting at $b$ yields that the ruin probability starting at $b$ is 1 , and thus the ruin probability starting at $u$ is 1 .

In the case that $\psi_{1}^{\prime}(0)>0$, i.e., $\Phi_{1}(0)>0$, (4.8) yields that $\mathcal{L}_{\mathcal{B}, \mathcal{D}}(0,0)<1$. Substituting (4.8) in (4.5) and solving for $u=b$ and noticing that $W^{(0)}(0)=1$ yields that the ruin probability starting at $b$ is

$$
\frac{\frac{1}{W^{(0)}(b)}}{1-\int_{0}^{b} \int_{z=y}^{\infty} u^{(0)}(0, y) \lambda d F_{Y}(z) e^{-\lambda(z-y-I(z-y))\left(1-\mathcal{L}_{Y-I(Y)}\left(\Phi_{1}(0)\right)\right)} d y} .
$$

Substituting $\theta=\beta=0$ and (4.10) in (4.4) and (4.5) gives (4.6) and (4.7).

\subsection{The expected discounted dividends}

The analysis of the expected discounted dividends for general $I(x)$ is similar to the analysis for the case where $I(x)=(1-\alpha) x$. Let $\mathcal{V}^{(q)}(u, b)$ be the expected discounted dividends until $\mathcal{U}$ reaches $b$ given that $\mathcal{U}(0)=u$ as defined in Section 3.3, but where the dividend that is taken from a gain of size $x$ is $I(x)$, and $\mathcal{B}$ is the generic busy period in a $M / G / 1$ queue with arrival rate $\lambda$ and service time distributed as $Y-I(Y)$. Thus in the general case $\eta$ is defined as in (3.28) with $\mathcal{B}$ as defined above.

\section{Proposition 4.3.}

(i) Let $\mathcal{U}(0)=u=b+y, y>0$. Then

$\mathcal{V}^{(q)}(u, b)=I(y)+V(q) \frac{\lambda}{\eta}(1-\exp (-(y-I(y)) \eta))$,

where $V(q)=\mathbb{E}\left[\mathcal{V}^{(q)}(Y+b, b)\right]$ is given by

$$
V(q)=\frac{\mathbb{E}[I(Y)]}{1-\frac{\lambda}{\eta}(1-\mathbb{E}[\exp (-\eta(Y-I(Y)))])} .
$$

(ii) Let $\mathcal{U}(0)=u \leq b$ then

$$
\begin{aligned}
& \mathcal{V}^{(q)}(u, b) \\
& =\int_{y=0}^{b} \int_{z=y}^{\infty} u^{(q)}(b-u, y) \mathcal{V}^{(q)}(b+z-y, b) \lambda d F(z) d y,
\end{aligned}
$$$$
\text { where } \mathcal{V}^{(q)}(b+z-y, b) \text { is given by (4.11). }
$$

\section{Proof.}

(i) The proof of (4.11) is the same as the proof of (3.25).

(ii) (4.13) is straightforward from the definition of the potential measure $u^{(q)}(\cdot, \cdot)$ in (2.15).

Now we obtain the expected discounted dividends for the general case. The proof of the following proposition is similar to the proof of Proposition 3.7.

\section{Proposition 4.4.}

$\mathcal{V}_{\text {tot }}(u)=\mathcal{V}^{(q)}(u, b)+\frac{\delta_{u} \mathcal{V}^{(q)}(b, b)}{1-\delta_{b}}$, where

$\delta_{u}=e^{-\Phi_{1}(q)(u-b-I(u-b))}$

for $u>b$, and

$\delta_{u}=\int_{y=0}^{b} \int_{z=y}^{\infty} u^{(q)}(b-u, y) e^{-\Phi_{1}(q)(z-y-I(z-y))} \lambda d F(z) d y$

for $u \leq b$.

Proof. (4.14) is similar to (3.41). For $u>b$ the discount factor in (4.15) is obtained similarly to (3.38).

The discounted time until the process reaches $b$ from above when $\mathcal{U}(0)=u<b$ is

$\delta_{u}=\left[e^{-q\left(C_{u}+P_{u}\right)} 1_{C_{u}<H_{u}}\right]$

$=\mathbb{E}_{b-u}\left[e^{-q \tau_{0}^{-}} 1_{\tau_{0}^{-}<\tau_{b}^{+}} e^{\Phi_{1}(q)\left(X\left(\tau_{0}^{-}\right)-I\left(X\left(\tau_{0}^{-}\right)\right)\right)}\right]$.

To evaluate (4.17) we use the $q$-potential measure again:

$\delta_{u}=\int_{y=0}^{b} \int_{z=y}^{\infty} u^{(q)}(b-u, y) e^{-\Phi_{1}(q)(z-y-I(z-y))} \lambda d F(z) d y$.

The mean of the total discounted dividends is obtained as in (3.41).

Remark 4.1. Substituting $I(y)=(1-\alpha) y$ in (4.15) immediately yields (3.38). Substituting $I(y)=(1-\alpha) y$ in (4.16) gives

$\delta_{u}=\int_{y=0}^{b} \int_{z=y}^{\infty} u^{(q)}(b-u, y) e^{-\Phi_{1}(q) \alpha(z-y)} \lambda d F(z) d y$.

The definition of $u^{(q)}$ in (2.15) yields that the expression in (4.19) equals $E_{b-u}\left[e^{-q \tau_{0}^{-}} 1_{\tau_{0}^{-}<\tau_{b}^{+}} e^{\Phi_{1}(q) \alpha X\left(\tau_{0}^{-}\right)}\right]$as in (3.40).

\section{Acknowledgment}

The research of Onno Boxma was supported via a TOP-C1 grant of the Netherlands Organisation for Scientific Research.

\section{References}

Albrecher, H., Ivanovs, J., Zhou, X., 2016. Exit identities for Lévy processes observed at Poisson arrival times. Bernoulli 22 (3), 1364-1382.

Albrecher, H., Thonhauser, S., 2009. Optimality results for dividend problems in insurance. Rev. R. Acad. Cienc. Exactas Fs. Nat. Ser. A Mat. 103 (2), 295-320.

Avanzi, B., Gerber, H.U., Shiu, E.S.W., 2007. Optimal dividends in the dual risk model. Insurance Math. Econom. 41, 111-123.

Avanzi, B., 2009. Strategies for dividend distribution: A review. N. Am. Actuar. J. 13, 217-251.

Avanzi, B., Pérez, J.L., Wong, B., Yamazaki, K., 2017. On optimal joint reflective and refractive dividend strategies in spectrally positive Lévy models. Insurance Math. Econom. 72, 148-162.

Bayraktar, E., Kyprianou, A., Yamazaki, K., 2013. On the optimal dividends in the dual model. Astin Bull. 43 (3), 359-372.

Cheung, E.C.K., Wong, J.T.Y., 2017. On the dual risk model with Parisian implementation delays in dividend payments. European J. Oper. Res. 257, 159-173.

Cheung, E.C.K., Zhang, Z., 2018. Periodic threshold-type dividend strategy in the compound Poisson risk model. Scand. Actuar. J.

Egami, M., Yamazaki, K., 2014. Phase-type fitting of scale functions for spectrally negative Lévy processes. J. Comput. Appl. Math. 264, 1-22.

Kyprianou, A.E., 2006. Introductory Lectures on Fluctuations of Lévy processes with Applications. Springer, Berlin.

Landriault, D., Renaud, J.-F., Zhou, X., 2014. An insurance risk model with Parisian implementations delays. Methodol. Comput. Appl. Probab. 16 (3), 583-607.

$\mathrm{Ng}, \mathrm{A} ., 2009$. On the dual model with a dividend threshold. Insurance Math. Econom. $44,315-324$

Takács, L., 1962. Introduction to the Theory of Queues. Oxford University Press, New York.

Yin, C., Wen, Y., 2013. Optimal dividend problem with terminal value for spectrally positive Lévy processes. Insurance Math. Econom. 53 (3), 769-773.

Yin, C., Wen, Y., Zhao, Y., 2014. On the dividend problem for a spectrally positive Lévy process. Astin Bull. 44 (3), 635-651. 